\title{
Autocrine Production of Interleukin-6: A Mechanism of Interleukin-6 Independence in Dexamethasone-Resistant 7TD1 Murine Myeloma Cells
}

\author{
Kalyan J. Gangavarapu1,2*, Alok Bhushan ${ }^{3}$, James C. K. Lai1 ${ }^{1}$, Christopher K. Daniels ${ }^{1}$ \\ ${ }^{1}$ Department of Biomedical and Pharmaceutical Sciences, College of Pharmacy, The ISU Biomedical Research \\ Institute, Division of Health Sciences, Idaho State University, Pocatello, USA \\ ${ }^{2}$ Department of Pharmacology and Therapeutics, Roswell Park Cancer Institute, Buffalo, USA \\ ${ }^{3}$ Department of Pharmaceutical Sciences, Jefferson School of Pharmacy, Thomas Jefferson University, \\ Philadelphia, USA \\ Email: *kalyan.gangavarapu@roswellpark.org
}

Received 7 February 2014; revised 7 March 2014; accepted 14 March 2014

Copyright (C) 2014 by authors and Scientific Research Publishing Inc.

This work is licensed under the Creative Commons Attribution International License (CC BY).

http://creativecommons.org/licenses/by/4.0/

(c) () Open Access

\section{Abstract}

Several factors could contribute to proliferation of multiple myeloma (MM) cells independent of interleukin-6 (IL6) in the later stages of the disease. Our previous studies established a dexamethasone-resistant 7TD1 cell line (7TD1-Dxm) and have shown that one mechanism of resistance to dexamethasone is due to inhibition of cytochrome c release. We have also observed that 7TD1Dxm cells proliferate independently of externally-added IL6. This study therefore aimed to elucidate the mechanisms responsible for IL6-independent proliferation in 7TD1-Dxm cells. Our results indicated that 7TD1-Dxm cells produced IL6 in an autocrine fashion. We have observed that dexamethasone-resistant 7TD1 cells become dexamethasone-resistant and IL6-independent for proliferation concomitantly. This strongly suggests that production of IL6 by 7TD1-Dxm cells may play an important role in the development of dexamethasone resistance. Consequently, further investigation of the molecular mechanisms responsible for IL6 production may be helpful in delineating the mechanisms leading to dexamethasone resistance.

\section{Keywords}

Multiple Myeloma, Interleukin-6, 7TD1 Cells, Autocrine Production, Dexamethasone Resistance

\footnotetext{
${ }^{*}$ Corresponding author.
}

How to cite this paper: Gangavarapu, K.J., et al. (2014) Autocrine Production of Interleukin-6: A Mechanism of Interleukin-6 Independence in Dexamethasone-Resistant 7TD1 Murine Myeloma Cells. Journal of Cancer Therapy, 5, 523-530. 


\section{Introduction}

Multiple myeloma (MM) is a plasma cell neoplasm currently without a cure. Two components are believed to be important in promoting the malignancy of plasma cells: 1) the multi-step genetic alterations leading to the activation of several growth and cell survival signaling pathways and 2) the interactions between the malignant plasma cells (also referred to as myeloma cells) and the bone marrow stromal cells (BMSC) giving rise to the secretion of several chemokine and cytokine growth factors [1]-[5]. Adhesion of myeloma cells to BMSC is mediated by adhesion molecules like intracellular adhesion molecules (I-CAM), very late activating antigen-4 (VLA-4), vascular cell adhesion molecule-1 (VCAM-1), and lymphocyte function-associated antigen-1 (LFA-1) [6]-[8]. Of the several growth factors secreted due to the molecular interactions arising from adhesion of MM cells to BMSC, interleukin-6 (IL6) is a well-studied and characterized cytokine growth factor promoting proliferation in and conferring anti-apoptotic protection on myeloma cells [1] [2] [9]-[14].

Two major mechanisms in late stage MM are known to render such patients unresponsive to first-line chemotherapeutic agents (e.g., dexamethasone). 1) When myeloma cells start proliferating in the bone marrow microenvironment (BMM), they eventually become resistant to treatment with conventional chemotherapeutic agents like dexamethasone (Dxm) [9]-[14]. 2) The myeloma cells become IL6-independent for proliferation [12]-[14]. However, whether the patients’ MM cells develop dexamethasone resistance and IL6 independence concomitantly has not been clarified. Furthermore, whether chemotherapy resistance and IL6 independence in MM share common pathogenic mechanisms are also presently unknown.

Previous studies in our laboratory have established a dexamethasone-resistant 7TD1 sub-line (7TD1-Dxm) and have observed that 7TD1-Dxm cells show IL6 independence for proliferation and dexamethasone resistance concomitantly [15]. We have also shown that dexamethasone resistance observed in 7TD1-Dxm cells is due to inhibition of cytochrome c release [16]. This study therefore aimed to further elucidate mechanism(s) underlying the development of IL6 independence in 7TD1-Dxm cells.

\section{Methods}

\subsection{Cell Culture}

7TD1-Dxm (Dexamethasone-resistant 7TD1) cells were generated as described previously [15]-[19]. Both 7TD1 and 7TD1-Dxm cells were cultured and maintained in complete culture medium as noted previously [15][19]. Appropriate vehicle controls were included in experiments. Anti-IL6 antibody (Santa Cruz Biotechnology, Inc., Dallas, TX) was used at a concentration of $2 \mu \mathrm{g} / \mathrm{ml}$. Cellular proliferation was determined using the MTT assay [15]-[20]. All experimental procedures were carried out with a prior wash-out period of 24 hrs during which both the cell lines were incubated without IL6 or Dxm. All experiments utilized parent 7TD1 cells between passages 30 and 60 .

\subsection{Detection of Apoptosis}

Apoptotic cells were quantified using DNA fragmentation TUNEL assay (Molecular Probes, Eugene, OR). Briefly, 7TD1 and 7TD1-Dxm cells were subjected to various treatments, incubated for 48 hrs and processed as described previously [15]-[17] [19] before they were analyzed using a flow cytometer (Becton Dickinson, East Rutherford, NJ).

\subsection{Conditioned Medium Experiment}

7TD1-Dxm cells were incubated in the absence of $85 \mu \mathrm{M}$ dexamethasone or IL6 for 72 hrs. After that treatment, the medium from the 7TD1-Dxm cells so treated was collected and then employed to resuspend the parent 7TD1 cells and plated them in triplicates in a 96-well plate in the presence and absence of $2 \mu \mathrm{g} / \mathrm{ml}$ anti-IL6 antibody and incubated at $37^{\circ} \mathrm{C}$ for another 72 hrs. Subsequently, their proliferation in 72 hours was studied using the MTT assay [15]-[20].

\subsection{RNA Isolation and First-Strand cDNA Synthesis}

RNA isolation and cDNA synthesis were performed as described previously [19]. Briefly, both parent 7TD1 cells and 7TD1-Dxm cells were incubated in the absence of IL6 for 72 hrs and then RNA was isolated using the 
RNAqueous kit (Ambion, Grand Island, NY), according to the manufacturer's protocol. The concentration of total RNA was determined using a gene quant system. Total RNA $(4 \mu \mathrm{g})$ from each sample was reverse transcribed into single-stranded cDNA with a Cells-to-Signal kit using oligo (dT) primers. After incubation at $40^{\circ} \mathrm{C}$ for $2 \mathrm{~min}$ and at $40^{\circ} \mathrm{C}$ for $50 \mathrm{~min}$, M-MLVT reverse transcriptase was denatured at $70^{\circ} \mathrm{C}$ for $5 \mathrm{~min}$. The cDNA so obtained was stored at $-20^{\circ} \mathrm{C}$ until used for polymerase chain reaction (PCR).

\subsection{Polymerase Chain Reaction}

Polymerase chain reaction was carried out as described previously [19]. Briefly, the synthesized cDNA was amplified by PCR using a PCR core kit and Gene Amp PCR system 2400 Gradient thermal cycler (Perkin Elmer, Waltham, MA). PCR analyses for GAPDH, and IL6 were performed using PCR core kit standard protocol. Conditions for GAPDH and IL6 were: denaturing at $94^{\circ} \mathrm{C}$ for 3 min, annealing for $30 \mathrm{sec}$ at $62^{\circ} \mathrm{C}$ for GAPDH and at $52^{\circ} \mathrm{C}$ for IL6. The sequences of primers used were: GAPDH sense, 5'-ACCACAGTCCATGCCATCAC-3'; GAPDH antisense, 5'-TCCACCACCCTGTTGCTGTA-3'; IL6 sense 5'-CTGACAATATGAATGTTGGG-3'; and IL6 antisense, 5'-TCCAAGAAACCATCTGGCTAGG-3'. PCR products were electrophoretically sizefractionated in $1.5 \%$ agarose gel and visualized using ethidium bromide. The estimated sizes of the PCR products were: GAPDH, 452 bp and IL6, 200 bp.

\subsection{Statistical Analyses of Data}

Statistical significance of experimental results was analyzed by univariate analysis of variance followed by Tukey's post-hoc test with a minimum significance level set at $\mathrm{p}<0.05$. In figures, individual letters identify those treatment groups where the differences are statistically significant as indicated by ANOVA (e.g., all treatment groups identified by a letter "a" are statistically significantly different from those treatments identified by all other letters (i.e., "b”, “c”, etc.)).

\section{Results}

\subsection{Increasing Concentrations of Anti-IL6 Antibody Increased Its Inhibitory Effect on Proliferation of 7TD1-Dxm Cells}

Our previous studies [19] demonstrated that treatment with an anti-IL6 antibody decreased proliferation of 7TD1-Dxm cells, suggesting 7TD1-Dxm cells produced IL6 and utilized it for proliferation. Therefore, we used increasing concentrations $(0.02 \mu \mathrm{g} / \mathrm{ml}, 0.2 \mu \mathrm{g} / \mathrm{ml}, 1 \mu \mathrm{g} / \mathrm{ml}$, and $2 \mu \mathrm{g} / \mathrm{ml})$ of the anti-IL6 antibody to determine the minimum concentration of the antibody required to inhibit proliferation of 7TD1-Dxm cells. Figure 1 shows proliferation of 7TD1-Dxm cells was significantly inhibited by the anti-IL6 antibody only at $2 \mu \mathrm{g} / \mathrm{ml}$ (The cells were also incubated with an IgG antibody at $2 \mu \mathrm{g} / \mathrm{ml}$, which was used as a negative control). These findings suggested that dexamethasone-resistant 7TD1 cells might be producing IL6 and utilizing it.

\subsection{Conditioned Medium from 7TD1-Dxm Cells Promoted Proliferation of Parent 7TD1 Cells}

We examined the effect of conditioned medium from 7TD1-Dxm cells on proliferation of parent 7TD1 cells. 7TD1-Dxm cells were cultured in the absence of $85 \mu \mathrm{M}$ dexamethasone for $72 \mathrm{hrs}$. Parent 7TD1 cells were IL6 withdrawn for 24 hrs. After incubation for $24 \mathrm{hrs}$, 7TD1 cells were resuspended in $1 \mathrm{ml}$ of the conditioned medium from 7TD1-Dxm cells and then plated in triplicates at a concentration of $10^{4}$ cells $/ \mathrm{ml}$ in the presence or absence of $2 \mu \mathrm{g} / \mathrm{ml}$ anti-IL6 antibody. Figure 2 shows that the conditioned medium from 7TD1-Dxm cells promoted significant increase in proliferation of 7TD1 cells. This proliferation triggered by IL6 from conditioned medium was inhibited in the presence of $2 \mu \mathrm{g} / \mathrm{ml}$ anti-IL6 antibody. These findings suggested that 7TD1-Dxm cells are producing and utilizing IL6 for proliferation.

\subsection{Treatment with Anti-IL6 Antibody Alone or in Combination with Dexamethasone Did Not Induce Apoptosis in 7TD1-Dxm Cells}

To examine the effect of the anti-IL6 antibody on induction of apoptosis in 7TD1-Dxm cells, we treated both 


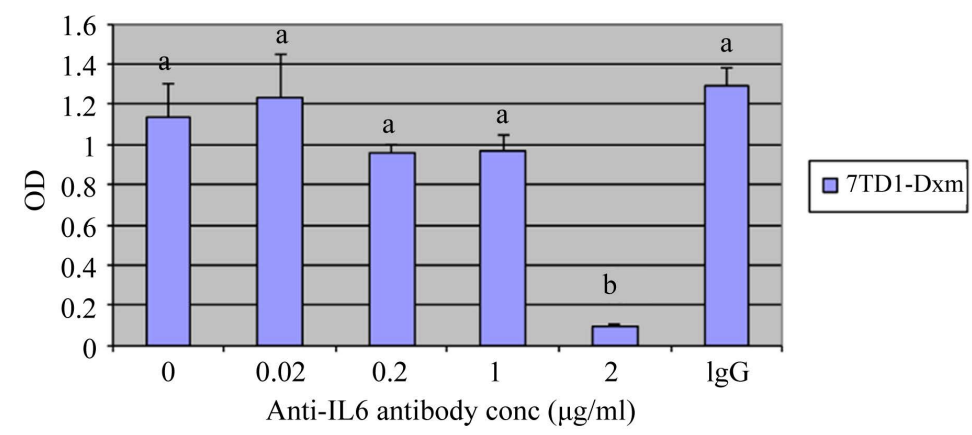

Figure 1. Effect of treatment of 7TD1-Dxm cells with increased concentrations of anti-IL6 antibody on their proliferation. 7TD1-Dxm cells were treated with increasing concentrations, $0.02 \mu \mathrm{g} / \mathrm{ml}, 0.2 \mu \mathrm{g} / \mathrm{ml}, 1 \mu \mathrm{g} / \mathrm{ml}$, and $2 \mu \mathrm{g} / \mathrm{ml}$ of anti-IL6 antibody in the absence of IL6. After $72 \mathrm{hrs}$ in culture, cell proliferation was determined using MTT assay. Values represent mean O.D. \pm S.D. for assays performed in three separate experiments with triplicates in each experiment. Treatment of 7TD1-Dxm cells with increased concentrations of anti-IL6 antibody increased its inhibitory effect on their proliferation with significant inhibition of proliferation observed at $2 \mu \mathrm{g} / \mathrm{ml}$.

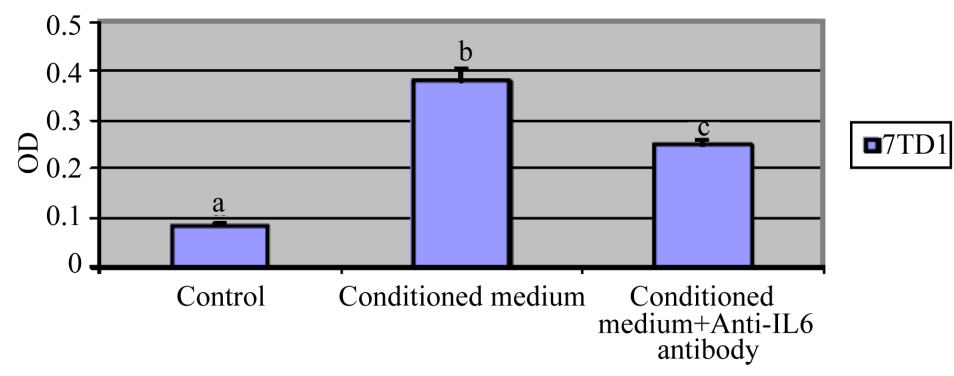

Figure 2. Effect of treatment with conditioned medium from 7TD1-Dxm cells on proliferation of parent 7TD1 cells. 7TD1-Dxm cells were cultured in the absence of dexamethasone for 72 hrs. Then medium from the cells was collected and parent 7TD1 cells were cultured in that conditioned medium in the presence and absence of $2 \mu \mathrm{g} / \mathrm{ml}$ anti-IL6 antibody. After 72 hrs in culture, cell proliferation was determined using the MTT assay. Values represent mean O.D. \pm S.D. for assays performed in three separate experiments with triplicates in each experiment. Conditioned medium from 7TD1-Dxm cells significantly increased proliferation of the parent 7TD1 cells and this proliferation was significantly inhibited in the presence of an anti-IL6 antibody.

parent 7TD1 and 7TD1-Dxm cells with $2 \mu \mathrm{g} / \mathrm{ml}$ of anti-IL6 antibody alone or in the presence of $85 \mu \mathrm{M}$ dexamethasone. Figure 3 shows that treatment of parent 7TD1 cells with anti-IL6 antibody alone or in the presence of $85 \mu \mathrm{M}$ dexamethasone induced apoptosis to a significant extent and the increase in the \% apoptotic cells was approximately 8 -fold (11\% apoptotic cells in the presence of anti-IL6 antibody alone and $80 \%$ when treated with both anti-IL6 antibody and $85 \mu \mathrm{M}$ dexamethasone). 7TD1-Dxm cells were quite resistant to induction of apoptosis when treated with anti-IL6 antibody alone or with both anti-IL6 antibody and $85 \mu \mathrm{M}$ dexamethasone. These results suggested that even though anti-IL6 antibody inhibited proliferation of the 7TD1-Dxm cells, it did not have any impact on induction of apoptosis and presence of dexamethasone with anti-IL6 antibody did not reverse the resistance of the cells to dexamethasone. Taken together, these observations suggested proliferation and resistance of 7TD1-Dxm cells appeared to be regulated by different mechanisms as their proliferation was inhibited by treatment with an IL6-neutralizing antibody but did not induce apoptosis nor did it reverse dexamethasone resistance in 7TD1-Dxm cells. On the other hand, the results shown in Figure 2 suggested the likelihood that 7TD1-Dxm cells were producing and utilizing IL6 in an autocrine fashion and the produced IL6 could exert a protective effect on the resistant cells against induction of apoptosis. 
TUNEL ASSAY

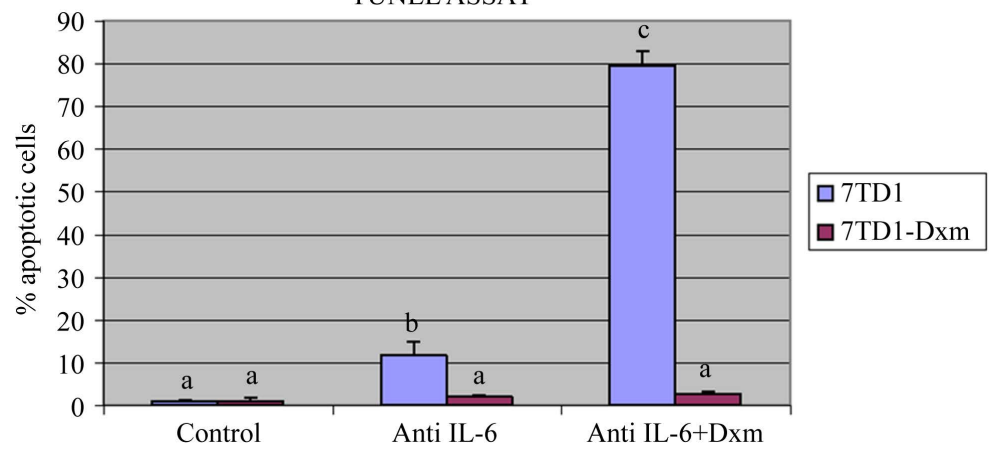

Figure 3. Effect of treatment with an anti-IL6 antibody on induction of apoptosis in 7TD1 and 7TD1-Dxm cells. Both parent 7TD1 and 7TD1-Dxm cells were treated with $2 \mu \mathrm{g} / \mathrm{ml}$ anti-IL6 antibody alone or in the presence of 85 $\mu \mathrm{M}$ dexamethasone and in the absence of IL6 at $37^{\circ} \mathrm{C}$ for $48 \mathrm{hrs}$. After treatment, the samples were fixed and subsequently subjected to TUNEL assay and the DNA fragmentation therein analyzed by flow cytometry. Values represent mean percentage apoptotic cells \pm S.D. for assays performed in two separate experiments with triplicates in each experiment. Parent 7TD1 cells showed a significant increase in apoptosis when treated with an anti-IL6 antibody alone or in the presence of $85 \mu \mathrm{M}$ dexamethasone unlike 7TD1-Dxm cells, which were resistant to induction of apoptosis in the presence of antiIL6 antibody alone or in the presence of $85 \mu \mathrm{M}$ dexamethasone.

\subsection{IL6 Gene Was Expressed in 7TD1-Dxm Cells}

The results discussed so far are consistent with the hypothesis that 7TD1-Dxm cells produce and use IL6 in an autocrine fashion. To seek further support of this hypothesis, we examined the expression of the IL6 gene in both 7TD1-Dxm and the parent 7TD1 cells. Both 7TD1 and 7TD1-Dxm cells were cultured in the absence of IL6 for 72 hrs. Subsequently, total RNA was isolated from both cell types following the manufacturer's protocol and then a two-step reverse transcription PCR (RTPCR) was performed according to the standard protocol for RTPCR as described previously [19]. Their respective PCR products were run on a 1.5\% agarose gel. GAPDH was used as a positive control. Figure 4 shows that 7TD1-Dxm cells expressed the IL6 gene unlike the parent 7TD1 cells, which did not express the IL6 gene in the absence of externally-added IL6. Thus, these findings provided additional support for our hypothesis that 7TD1-Dxm cells produce and utilize IL6 in an autocrine fashion.

\section{Discussion}

Development of resistance to treatment with chemotherapeutic agents like dexamethasone is a major problem in treatment of patients with MM. Nevertheless, the mechanisms that allow MM cells to develop resistance to such agents are far from being fully understood. For example, whether development of a dexamethasone-resistant and IL6-independent clone can result from the selective pressure to survive the continuous exposure to dexamethasone in the absence of IL6 remains to be elucidated, even though clonal heterogeneity is known to occur in patients with MM which provides the clones with characteristics necessary for survival [21] [22]. This study therefore aimed to address some of the key but as yet unresolved mechanistic issues.

MM cells may be dependent on IL6 for proliferation and survival in the intramedullary stage of the disease. But, once in the extramedullary stage, MM cells are in the blood circulation and IL6 may not be available to them. This scenario strongly suggests that there is a point between the intramedullary and extramedullary stages of the disease where MM cells develop IL6-independence and probably resistance to treatment with chemotherapeutic agents. Consequently, development of sub-clones of MM cells insensitive to drug treatment and proliferating independent of IL6 may occur at this juncture. Therefore, in this study, we have investigated the novel hypothesis that the IL6-independent proliferation and resistance to chemotherapeutic agent such as dexamethasone in MM cells are regulated by different mechanisms.

We [15]-[19] and others [23]-[28] have elucidated some of the mechanisms underlying the IL6-independent 


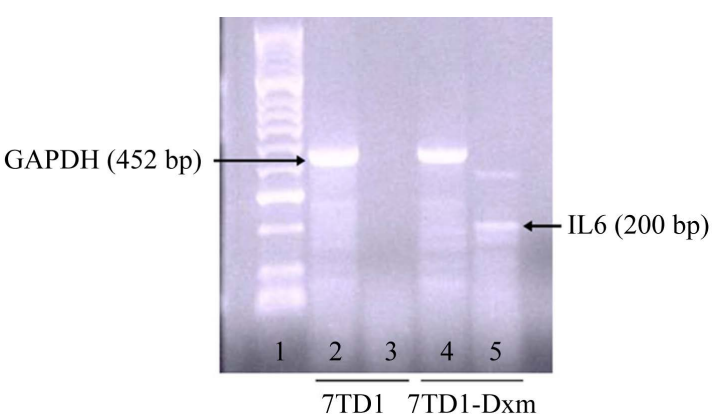

\begin{abstract}
Figure 4. Expression of interleukin-6 in 7TD1 and 7TD1Dxm cells. Both parent 7TD1 and 7TD1-Dxm cells were cultured in the absence of externally-added IL6. After 72 hrs, total RNA was isolated from the two cell types following the manufacturer's protocol and then two-step RTPCR was performed on them according to the standard protocol for RTPCR. Their respective PCR products were run on a $1.5 \%$ agarose gel and photographed. GAPDH was used as a positive control. Lanes 2 and 4 represent expression of GAPDH in total RNA from 7TD1 and 7TD1-Dxm cells, respectively, both cultured in the absence of externallyadded IL6. Lanes 3 and 5 represent expression of IL6 gene in total RNA from 7TD1 and 7TD1-Dxm cells, respectively, both cultured in the absence of externally-added IL6. 7TD1-Dxm cells expressed IL6 gene even in the absence of externally-added IL6 unlike the parent 7TD1 cells, which did not express IL6 gene in the absence of exogenous IL6.
\end{abstract}

proliferation of MM cells. There is a consensus in favor of the hypothesis that MM cells are producing and utilizing IL6 in an autocrine fashion and the produced IL6 can exert a protective effect on the chemo-resistant cells against induction of apoptosis. We found that: 1) treatment of the dexamethasone-resistant 7TD1-Dxm cells with an IL6-neurtalizing antibody led to inhibition of their proliferation at the antibody concentration of $2 \mu \mathrm{g} / \mathrm{ml}$ (Figure 1) and 2) treatment of the parent 7TD1 cells with conditioned medium from the dexamethasone-resistant 7TD1-Dxm cells promoted proliferation of 7TD1 cells (Figure 2). These findings provide some support for the hypothesis that MM cells are producing and utilizing IL6 in an autocrine fashion. Indeed, other investigators have also observed autocrine IL6 secretion by MM cells [13] [23].

Several mechanisms have been noted to account for autocrine IL6 secretion by MM cells leading to their continued survival and proliferation. Jourdan et al. concluded that a small IL6 autocrine production by MM cells was sufficient to trigger their cell cycling [25]. Bohnhorst et al. demonstrated that MM cells in the tumor environment express more toll-like receptors, which mediate their proliferation and survival partially due to autocrine production of IL6 [26]. Recent evidence points to constitutive NF- $\mathrm{BB}$ (nuclear factor kappa-light-chain-enhancer of activated B cells) activation and up-regulation of anti-apoptotic genes and cytokine receptors, all of which are related to MM disease progression and MM cell biology, and are involved in the development of an IL6-independent clone of MM cells [27].

In addition to providing evidence for the hypothesis that MM cells are producing and utilizing IL6 in an autocrine fashion, our results also demonstrated even though anti-IL6 antibody inhibited proliferation of 7TD1Dxm cells, it did not affect induction of apoptosis nor did it reverse the resistance of these cells to dexamethasone in the presence of dexamethasone (Figure 3). Furthermore, we found that the dexamethasone-resistant 7TD1Dxm cells, but not their parent 7TD1 cells, expressed the IL6 gene in the absence of externally-added IL6 (Figure 4). Thus, taken together, the results of this study not only are consistent with the notion that MM cells are producing and utilizing IL6 in an autocrine fashion but also provide some support for the hypothesis that the IL6-independent proliferation and resistance to chemotherapeutic agent such as dexamethasone in MM cells are regulated by different mechanisms.

In contrast to the mechanisms that lead to the IL6-independent proliferation of MM cells, the mechanisms that 
give rise to resistance to dexamethasone are much less well understood. Our previous studies and our current findings (e.g., Figure 3) have provided some insights into such mechanisms. We noted that STAT3 is constitutively activated in 7TD1-Dxm cells but the development of resistance to dexamethasone is downstream of the IL6 induced JAK/STAT3 pathway [15] and IL6 and JAK2/STAT3 signaling mediate the reversal of resistance to dexamethasone after withdrawal of dexamethasone in 7TD1-Dxm cells for over 3 - 5 months [17]. In particular, withdrawal of dexamethasone for over 3 or more months can restore the sensitivity of 7TD1-Dxm cells to inhibition of proliferation and induction of apoptosis close to that of the parent 7TD1 cells [17]. Furthermore, we also found that regulation in cytochrome c release from mitochondria differs between 7TD1 and 7TD1-Dxm cells [16]. Cytochrome c release is inhibited in 7TD1-Dxm cells leading to resistance to dexamethasone induced apoptosis in 7TD1-Dxm cells [16]. More recently, two other groups [27] [28] have reported findings that led them to make conclusions similar to and/or compatible with ours as discussed above.

Yang et al. observed that constitutive activation of NF- $\mathrm{BB}$ protected murine plasmacytoma cells from induction of apoptosis and also conferred IL6 independence for proliferation on those cells [27]. They further noted that IL6 independence and resistance are independent of the JAK/STAT pathway and the protective effect against IL6 withdrawal-induced apoptosis is due to the constitutive NF- $\kappa$ B activation and blockage of caspase activation and not due to IL6 production [27]. Similarly, Verdelli et al. found that IL6 independence demonstrated by a CMA03/06 sub-clone is in part attributable to NF- $\mathrm{B}$ activation and up-regulation of anti-apoptotic genes [28]. Furthermore the CMA-03/06 sub-clones have shown higher sensibility to NF- $\kappa$ B inhibition by bortezomib indicating that NF- $\mathrm{KB}$ signaling pathway plays a major role in conferring IL6 independence in CMA-03/06 sub-clones [28]. Results from our current study indicated that 7TD1-Dxm cells produce IL6. Based on previous studies [27] [28] and results from our current study, further investigation using bortezomib followed by dexamethasone treatment may be helpful in sensitizing the 7TD1-Dxm cells to dexamethasone induced apoptosis by overcoming IL6 independence.

The results of this study, together with some of those in our previous studies [15]-[17] [19], have provided strong evidence supporting the hypothesis that MM cells are producing and utilizing IL6 in an autocrine fashion. We have also discussed the evidence that is consistent with the hypothesis that the IL6-independent proliferation and resistance to chemotherapeutic agent such as dexamethasone in MM cells are regulated by different mechanisms. Clearly further studies to unravel these mechanisms may have important therapeutic and related translational implications in advancing the treatment of MM.

\section{Acknowledgements}

This study was supported by NIH Grant P20RR16454 from the INBRE program of the National Center for Research Resources.

\section{References}

[1] Hallek, M., Bergsagel, L. and Anderson, K.C. (1998) Multiple Myeloma: Increasing Evidence for a Multistep Transformation Process. Blood, 91, 3-21.

[2] Klein, B., Zhang, X.G., Lu, Z.H. and Bataille, R. (1995) Interleukin-6 in Human Multiple Myeloma. Blood, 85, 863872.

[3] Hirano, T. (1991) Interleukin 6 (IL-6) and Its Receptor: Their Role in Plasma Cell Neoplasias. International Journal of Cell Cloning, 9, 166-184. http://dx.doi.org/10.1002/stem.5530090303

[4] Bommert, K., Bargou, R.C. and Stuhmer, T. (2006) Signalling and Survival Pathways in Multiple Myeloma. Europeam Journal of Cancer, 42, 1574-1580. http://dx.doi.org/10.1016/j.ejca.2005.12.026

[5] Fonseca, R., Barlogie, B., Bataille, R., Bastard, C., Bergsagel, P.L., Chesi, M., et al. (2004) Genetics and Cytogenetics of Multiple Myeloma: A Workshop Report. Cancer Research, 64, 1546-1558. http://dx.doi.org/10.1158/0008-5472.CAN-03-2876

[6] Bruno, B., Rotta, M., Giaccone, L., Massaia, M., Bertola, A., Palumbo, A. and Boccadoro, M. (2004) New Drugs for Treatment of Multiple Myeloma. Lancet Oncology, 5, 430-442. http://dx.doi.org/10.1016/S1470-2045(04)01511-6

[7] Hideshima, T., Chauhan, D., Richardson, P. and Anderson, K.C. (2005) Identification and Validation of Novel Therapeutic Targets for Multiple Myeloma. Journal of Clinical Oncology, 23, 6345-6350. http://dx.doi.org/10.1200/JCO.2005.05.024

[8] Kim, I., Uchiyama, H., Chauhan, D. and Anderson, K.C. (1994) Cell Surface Expression and Functional Significance 
of Adhesion Molecules on Human Myeloma-Derived Cell Lines. British Journal of Haematology, 87, 483-493. http://dx.doi.org/10.1111/j.1365-2141.1994.tb08302.x

[9] Potter, M. (2003) Neoplastic Development in Plasma Cells. Immunological Reviews, 194, 177-195. http://dx.doi.org/10.1034/j.1600-065X.2003.00061.x

[10] Lauta, V.M. (2003) A Review of the Cytokine Network in Multiple Myeloma: Diagnostic, Prognostic, and Therapeutic Implications. Cancer, 97, 2440-2452. http://dx.doi.org/10.1002/cncr.11072

[11] Hardin, J., MacLeod, S., Grigorieva, I., Chang, R., Barlogie, B., Xiao, H., et al. (1994) Interleukin-6 Prevents Dexamethasone-Induced Myeloma Cell Death. Blood, 84, 3063-3070.

[12] Chatterjee, M., Honemann, D., Lentzsch, S., Bommert, K., Sers, C., Herrmann, P., et al. (2002) In the Presence of Bone Marrow Stromal Cells Human Multiple Myeloma Cells Become Independent of the IL-6/gp130/STAT3 Pathway. Blood, 100, 3311-3318. http://dx.doi.org/10.1182/blood-2002-01-0102

[13] Frassanito, M., Cusmai, A., Iodice, G. and Dammacco, F. (2001) Autocrine Inteleukin-6 Production and Highly Malignant Multiple Myeloma: Relation with Resistance to Drug-Induced Apoptosis. Blood, 97, 483-489. http://dx.doi.org/10.1182/blood.V97.2.483

[14] Kyle, R.A. (2001) Management of Patients with Multiple Myeloma: Emphasizing the Role of High-Dose Therapy. Clinical Lymphoma, 2, 21-28. http://dx.doi.org/10.3816/CLM.2001.n.008

[15] Gangavarapu, K.J., Olbertz, J.L., Bhushan, A., Lai, J.C.K. and Daniels, C.K. (2008) Apoptotic Resistance Exhibited by Dexamethasone-Resistant Murine 7TD1 Cells Is Controlled Independently of Interleukin-6 Triggered Signaling. Apoptosis, 13, 1394-1400. http://dx.doi.org/10.1007/s10495-008-0265-y

[16] Gangavarapu, K.J., Bhushan, A., Lai, J.C.K. and Daniels, C.K. (2013) Differential Regulation of Cytochrome c Release in Dexamethasone-Resistant 7TD1 Cells. Journal of Cancer Therapy, 4, 835-842. http://dx.doi.org/10.4236/jct.2013.44095

[17] Liu, T., Fei, Z., Gangavarapu, K.J., Agbenowu, S., Bhushan, A., Lai, J.C.K., et al. (2013) Interleukin-6 and JAK2/ STAT3 Signaling Mediate the Reversion of Dexamethasone Resistance after Dexamethasone Withdrawal in 7TD1 Multiple Myeloma Cells. Leukemia Research, 37, 1322-1328. http://dx.doi.org/10.1016/j.leukres.2013.06.026

[18] Irvin, B.J., Hanson, C.L., Smith, L.H., Daniels, C.K. (2001) Cyclic AMP- and IL6-Signaling Cross Talk: Comodulation of Proliferation and Apoptosis in the 7TD1 B Cell Hybridoma. Experimental Cell Research, 265, 73-79. http://dx.doi.org/10.1006/excr.2001.5157

[19] Gangavarapu, K.J. (2008) Characterization of a Dexamethasone-Resistant 7TD1 Murine Myeloma Cell Line. Ph.D. Dissertation, Idaho State University, Pocatello.

[20] Mosmann, T. (2001) Rapid Colorimetric Assay for Cellular Growth and Survival: Application to Proliferation and Cytotoxicity Assays. Journal of Immunological Methods, 65, 55-63. http://dx.doi.org/10.1016/0022-1759(83)90303-4

[21] Egan, J.B., Shi, C.X., Tembe, W., Christoforides, A., Kurdoglu, A., Sinari, S., et al. (2012) Whole Genome Sequencing of Multiple Myeloma from Diagnosis to Plasma Cell Leukemia Reveals Genomic Initiating Events, Evolution and Clonal Tides. Blood, 120, 1060-1066. http://dx.doi.org/10.1182/blood-2012-01-405977

[22] Keats, J.J., Chesi, M., Egan, J.B., Garbitt, V.M., Palmer, S.E., Braggio, E., et al. (2012) Clonal Competition with Alternating Dominance in Multiple Myeloma. Blood, 120, 1067-1076. http://dx.doi.org/10.1182/blood-2012-01-405985

[23] Kawano, M., Hirano, T., Matsuda, T., Taga, T., Horii, Y., Iwato, K., et al. (1988) Autocrine Generation and Requirement of BSF-2/IL-6 for Human Multiple Myelomas. Nature, 332, 83-85. http://dx.doi.org/10.1038/332083a0

[24] Klein, B., Zhang, X.G., Jourdan, M., Content, J., Houssiau, F., Aarden, L., et al. (1989) Paracrine Rather Than Autocrine Regulation of Myeloma Cell Growth and Differentiation by Interleukin-6. Blood, 73, 517-523.

[25] Jourdan, M., Mahtouk, K., Veyrune, J.L., Couderc, G., Fiol, G., Redal, N., et al. (2005) Delineation of the Roles of Paracrine and Autocrine Interleukin-6 (IL-6) in Myeloma Cell Lines in Survival versus Cell Cycle. A Possible Model for the Cooperation of Myeloma Cell Growth Factors. European Cytokine Network, 16, 57-64.

[26] Bohnhorst, J., Rasmussen, T., Moen, S.H., Flottum, M., Knudsen, L., Borset, M., et al. (2006) Toll-Like Receptors Mediate Proliferation and Survival of Multiple Myeloma Cells. Leukemia, 20, 1138-1144. http://dx.doi.org/10.1038/sj.leu.2404225

[27] Yang, Y., Groshong, J.S., Matta, H., Gopalakrishnan, R., Yi, H. and Chaudhary, P.M. (2011) Constitutive NF-kappaB Activation Confers Interleukin 6 (IL6) Independence and Resistance to Dexamethasone and Janus Kinase Inhibitor INCB018424 in Murine Plasmacytoma Cells. Journal of Biological Chemistry, 286, 27988-27997. http://dx.doi.org/10.1074/jbc.M110.213363

[28] Verdelli, D., Nobili, L., Todoerti, K., Mosca, L., Fabris, S., D’Anca, M., et al. (2014) Molecular events Underlying Interleukin-6 Independence in a Subclone of the CMA-03 Multiple Myeloma Cell Line. Genes Chromosomes Cancer, 53, 154-167. http://dx.doi.org/10.1002/gcc.22127 